\title{
Epidural Anesthesia Combined with Intravenous Dexmedetomidine Sedation for Elective Ileostomy Closure in a Patient with Bilateral Lung Transplantation: A Case Report
}

\author{
Jacques de Montblanc, $M D^{1^{*}}$, Jamil Elmawieh, $M D^{1}$, Camille Tilly, $M D^{2}$ and Dan Benhamou, $M D^{1}$
}

${ }^{1}$ Service d'Anesthésie-Réanimation Chirurgicale, Hôpitaux Universitaires Paris-Sud (Assistance Publique - Hopitaux de Paris), Hôpital de Bicêtre, Le Kremlin Bicêtre, France

${ }^{2}$ Service de Chirurgie Digestive, Hôpitaux Universitaires Paris-Sud (Assistance Publique - Hopitaux de Paris), Hôpital de Bicêtre, Le Kremlin Bicêtre, France

\begin{abstract}
A patient who had undergone bilateral lung transplantation surgery four years ago and had significant residual pulmonary insufficiency was scheduled for elective ileostomy closure. The procedure was performed under epidural anesthesia, intravenous sedation with dexmedetomidine and spontaneous ventilation without tracheal intubation. The combination provided high quality intraoperative analgesia and a moderate level of sedation without causing respiratory depression or excessive hemodynamic instability. The pharmacological properties of dexmedetomidine contributed to make this technique safe and effective.
\end{abstract}

Keywords

Epidural anesthesia, Dexmedetomidine, lleostomy, Lung transplantation

\section{Introduction}

We present the case of dexmetedomidine sedation associated with epidural anesthesia in spontaneous ventilation without tracheal intubation for the closure of an elective ileostomy in a woman with a history of bilateral lung transplantation. The simultaneous use of regional anesthesia with dexmedetomidine sedation may lead to a decrease in morphine requirement and limitation of its respiratory adverse effects.

Written informed consent for case publication was obtained from the patient.

\section{Case Presentation}

A 32-year-old, $185 \mathrm{~cm}, 75 \mathrm{~kg}$, woman with a history of bilateral lung transplantation for pulmonary fibrosis complicated by pulmonary hypertension four years ago was scheduled for elective ileostomy closure. She had undergone small bowel resection and double ileostomy via median laparotomy after a flange obstruction two years ago. She had pulmonary graft dysfunction with bronchiolitis obliterans, her pulmonary function test were altered (FEV1 $=16 \%$ of predicted; FEF $25 / 75=6 \%$ of predicted; TLC $=109 \%$ of predicted). She also had Raynaud's syndrome. She was not oxygen-dependent and was able to climb one flight of stairs. During the anes- thesia interview, the patient also reported morphine intolerance (history of postoperative nausea and vomiting). Daily treatment included cyclosporine, mycophenolate mofetil, prednisone, salbutamol, nicardipine and loperamide. The patient was classified ASA Physical status IV. The patient consented to have her elective surgery performed in supine position with regional anesthesia. The expected duration of the surgical procedure was 60 minutes. Preoperative laboratory tests showed: hemoglobin $=11.3 \mathrm{~g} / \mathrm{dL}$, platelet count $=249$ $\mathrm{G} / \mathrm{l}, \mathrm{K}=4.3 \mathrm{mmol} / \mathrm{l}$, creatininemia $=100 \mu \mathrm{mol} / \mathrm{l}$, calculated glomerular filtration rate $=60 \mathrm{ml} / \mathrm{min}$. Preoperative venous

*Corresponding author: Jacques de Montblanc, MD, Service d'Anesthésie-Réanimation Chirurgicale, Hôpitaux Universitaires Paris-Sud (Assistance Publique - Hopitaux de Paris), Hôpital de Bicêtre, 78, rue du Général Leclerc, 94275, Le Kremlin Bicêtre, France, Tel: +33-14-521-3441

Accepted: June 22, 2019

Published online: June 24, 2019

Citation: de Montblanc J, Elmawieh J, Tilly C, et al. (2019) Epidural Anesthesia Combined with Intravenous Dexmedetomidine Sedation for Elective lleostomy Closure in a Patient with Bilateral Lung Transplantation: A Case Report. J Clin Anesth Pain Manag 3(1):79-81 
Citation: de Montblanc J, Elmawieh J, Tilly C, et al. (2019) Epidural Anesthesia Combined with Intravenous Dexmedetomidine Sedation for Elective lleostomy Closure in a Patient with Bilateral Lung Transplantation: A Case Report. J Clin Anesth Pain Manag 3(1):79-81

blood gases obtained while breathing room air showed the following: $\mathrm{pH}=7.30, \mathrm{PCO}_{2}=52 \mathrm{~mm} \mathrm{Hg}, \mathrm{PO}_{2}=30 \mathrm{mmHg}, \mathrm{HCO}_{3}$ $=25 \mathrm{mmol} / \mathrm{l}, \mathrm{SvO}_{2}=50 \%$. The pre anesthesia $\mathrm{SpO}_{2}$ value was not available because of Raynaud's syndrome. Following application of routine monitors ( $\mathrm{ECG}, \mathrm{NIBP}, \mathrm{SpO}_{2}$, nasal $\mathrm{EtCO}_{2}$ ) in the operating room, antibiotic prophylaxis was administered in accordance with the local protocol. After local anesthesia, an epidural catheter was placed at the T8-T9 level and $6 \mathrm{~cm}$ of the catheter were inserted in the epidural space. Anesthesia was established with $20 \mathrm{ml}$ lidocaine $20 \mathrm{mg} / \mathrm{ml}$ with $1 / 200.000$ epinephrine administered in divided aliquots after a negative test dose of $3 \mathrm{ml}$ of the same solution. The upper limit of loss of sensation to cold extended up to T4 level. A continuous dexmedetomidine infusion was started at $40 \mu \mathrm{g} / \mathrm{h}(0.5 \mu \mathrm{g} /$ $\mathrm{kg} / \mathrm{h})$ and titrated $(0.5-0.8 \mu \mathrm{g} / \mathrm{kg} / \mathrm{h})$ to maintain adequate sedation (Ramsay sedation score maintained between 3 and 4). During surgery, a continuous norepinephrine infusion of 0.02 to $0.08 \mu \mathrm{g} / \mathrm{kg} / \mathrm{min}$ was established to maintain mean arterial pressure above $75 \mathrm{mmHg}$. Paracetamol $1 \mathrm{gm}$ and nefopam 40 mg were given intravenously during surgery. Intravenous ondansetron $4 \mathrm{mg}$ and droperidol $0.625 \mathrm{mg}$ were administered to prevent nausea and vomiting. The surgery was performed via an elective incision around the stoma. The two ileal segments were joined by a manual anastomosis, which was then returned inside the abdomen. The abdominal wall was closed with separate stitches, and then the skin was closed. The total duration of the surgery was 58 minutes. At the end of the surgical procedure, both dexmedetomidine and norepinephrine infusions were stopped (see Table 1 for details). Two separate boluses of $5 \mathrm{ml}$ of lidocaine $20 \mathrm{mg} / \mathrm{ml}$ with epinephrine were administered 50 minutes after the first bolus for postoperative analgesia before leaving the operating room. An epidural infusion of $6 \mathrm{ml} / \mathrm{h}$ with plain ropivacaine $2 \mathrm{mg} / \mathrm{mL}$ was started in the PACU and administered during the next 24 hours. Because of morphine intolerance, no opioid was used either intravenously or epidurally but regular intravenous administration of paracetamol was added to the epidural infusion. The patient was discharged from the PACU and returned to the ward. Members of the acute pain service visited her regularly during the first few days after surgery. The patient experienced some pain on movement ( 5 on a 0-10 numerical scale) on Day 1 , but thereafter pain remained at less than 2 at rest or on movement. The patient did not experience any shivering episode during the surgical procedure or in the PACU. On the evening of Day 0 , the patient was allowed to drink water and was discharged home on Day 3. One month after surgery, the patient had not experienced any complication or pain.

\section{Discussion}

Elective ileostomy closure is a very common procedure in both the general population and transplanted subjects. Post lung transplantation bronchiolitis obliterans affects 50 to $60 \%$ of these patients $[1,2]$. Mechanical ventilation during anesthesia of lung transplant patients with bronchiolitis obliterans may be difficult because of intraoperative airway obstruction. In addition, postoperative weaning from the ventilator may be difficult to predict. Hypotension may be the result of chronic post-transplant adrenal suppression with altered stress response [1,2].

Regional anesthesia might be an alternative to general anesthesia in patients with severe pulmonary impairment [3] and in patients with previous lung transplant [1,2] in whom additional risks, such as tracheal stenosis or pulmonary vascular overload may be present. In case of abdominal surgery, general anesthesia is usually chosen even though it carries its own risks, such as pulmonary atelectasis. Because of the severely altered preoperative pulmonary function tests [4] and because the procedure was not planned to be long lasting, we considered that the surgical procedure could be safely performed under regional anesthesia combined with sedation. Dexmedetomidine is a highly selective alpha2-adrenoceptor agonist which provides anxiolysis and cooperative sedation without respiratory depression and significantly reduces the use of analgesic drugs. Coskuner, et al. studied the effects of an intravenous infusion of dexmedetomidine on the duration of anesthesia and the state of wakefulness in patients with an epidural bupivacaine block. They reported the increase of the duration of epidural anesthesia, the sedation and rare side-effects after administration of intravenous dexmedetomidine at $1 \mu \mathrm{g} / \mathrm{kg}$ as a loading dose followed by continuous administration at a dose of $0.4 \mu \mathrm{g} / \mathrm{kg} / \mathrm{h}$ [5]. $\mathrm{Ng}$, et al. reported the use of a combined intravenous infusion of dexmedetomidine and epidural anesthesia in five patients who underwent MRI procedures [6]. Iwata, et al. reported the anesthetic management of non-intubated video-assisted thoracoscopic surgery using epidural anesthesia and dexmedetomidine in three patients with severe respiratory dysfunction [7]. Dexmedetomidine is also expected to lower postoperative pain intensity and morphine requirements $[8,9]$ which was especially useful in this patient who highlighted her intolerance to morphine. In addition, dexmedetomidine and epidural anesthesia-induced postoperative opioid sparing is beneficial in these patients

Table 1: Main hemodynamic data.

\begin{tabular}{|l|l|l|l|l|l|l|}
\hline & $\begin{array}{l}\text { Pre- } \\
\text { anesthesia }\end{array}$ & $\begin{array}{l}\text { Beginning of } \\
\text { anesthesia }\end{array}$ & $\begin{array}{l}\text { Surgical } \\
\text { incision }\end{array}$ & Lowest NIBP & End of surgery & $\begin{array}{l}\text { PACU } \\
\text { discharge }\end{array}$ \\
\hline NIBP $(\mathrm{mm} \mathrm{Hg})$ & $126 / 093$ & $150 / 090$ & $100 / 070$ & $100 / 065$ & $140 / 090$ & $119 / 099$ \\
\hline $\mathrm{HR}(\mathrm{bpm})$ & 90 & 100 & 90 & 85 & 100 & 93 \\
\hline $\mathrm{SpO}_{2}(\%)$ & $(*)$ & 100 & 100 & 100 & 100 & 100 \\
\hline Dexmedetomidine $(\mu \mathrm{g} / \mathrm{kg} / \mathrm{h})$ & & 0.5 & 0.8 & 0.8 & 0.5 & stopped \\
\hline Norepinephrine $(\mu \mathrm{g} / \mathrm{kg} / \mathrm{min})$ & 0 & 0 & 0.02 & 0.08 & stopped \\
\hline
\end{tabular}

NIBP: non invasive blood pressure; HR: heart rate; ( $\left.{ }^{*}\right)$ : The pre anesthesia $\mathrm{SpO}_{2}$ value was not available because of Raynaud's syndrome. 
Citation: de Montblanc J, Elmawieh J, Tilly C, et al. (2019) Epidural Anesthesia Combined with Intravenous Dexmedetomidine Sedation for Elective Ileostomy Closure in a Patient with Bilateral Lung Transplantation: A Case Report. J Clin Anesth Pain Manag 3(1):79-81

and may protect them against the negative effects on bronchociliary clearance, reduction of cough reflex and reduced ventilatory response to hypercapnia [1]. As NSAIDS are also to be avoided due to the risk of impaired renal function, the postoperative use of epidural analgesia was thought to be beneficial and provided overall excellent analgesia.

Dexmedetomidine was also expected to lower the incidence of postoperative nausea and vomiting [9] and the incidence of shivering [10] with its associated cardiopulmonary risks [11-13]. In the present case, the surgical procedure was successfully carried out under regional anesthesia associated with sedation without any adverse event.

Careful consideration should be given to the placement of any invasive monitor [2]. Because of her Raynaud syndrome and the small size of her radial arteries, and also because surgical procedure was a relatively minor one, we chose to avoid invasive blood pressure monitoring. Therefore, cardiac output monitoring by a pulse contour technique could not be used. We also avoided placing an oesophageal Doppler in a non-sedated patient. Other non-invasive cardiac output monitoring techniques (ClearSight ${ }^{\mathrm{TM}}$ or Nexfin $^{\mathrm{TM}}$ ) were unavailable on the day of surgery.

Because weaning from the norepinephrine infusion was rapid and easy and considering both hemodynamic and respiratory stability and satisfactory analgesia, we decided to transfer the patient in the surgical ward after prolonged observation of several hours in the PACU. Postoperative pain was followed by the acute pain service. Oral feeding was rapidly increased according to the local protocol.

In conclusion, we describe for an elective ileostomy closure case, the feasibility of a combined use of IV sedation with dexmedetomidine and epidural anesthesia in a patient with a previous bilateral lung transplantation and subsequent severely depressed respiratory function. The moderately invasive type of surgery simplified the choice of anesthetic management but the overall strategy led to early discharge and rapid recovery of this very frail patient.

\section{Conflicts of Interests/Financial Disclosures}

None.

\section{References}

1. Feltracco P, Falasco G, Barbieri S, et al. (2011) Anesthetic considerations for nontransplant procedures in lung transplant patients. J Clin Anesth 23: 508-516.

2. Hammel L, Sebranek J, Hevesi Z (2010) The Anesthetic Management of Adult Patients with Organ Transplants Undergoing Nontransplant Surgery. Advances in Anesthesia 28: 211-244.

3. Savas JF, Litwack R, Davis K, et al. (2004) Regional anesthesia as an alternative to general anesthesia for abdominal surgery in patients with severe pulmonary impairment. Am J Surg 188: 603-605.

4. Ciprandi G, Capasso M, Tosca M, et al. (2012) A forced expiratory flow at $25-75 \%$ value $<65 \%$ of predicted should be considered abnormal: a real-world, cross-sectional study. Allergy and Asthma Proceedings 33: e5-e8.

5. Coskuner I,Tekin M, Katil, etal. (2007) Effects of dexmedetomidine on the duration of anesthesia and wakefulness in bupivacaine epidural block. Eur J Anaesthesiol 24: 535-540.

6. Ng AT, Ip-Yam PC (2011) Epidural anesthesia with intravenous dexmedetomidine sedation in the successful anesthetic management of MRI-guided focused ultrasound ablation of early prostatic cancer. J Anesth 25: 756-759.

7. Iwata $Y$, Hamai $Y$, Koyama $T$ (2016) Anesthetic management of nonintubated video-assisted thoracoscopic surgery using epidural anesthesia and dexmedetomidine in three patients with severe respiratory dysfunction. J Anesth 30: 324-327.

8. Naik Bl, Nemergut EC, Kazemi A, et al. (2016) The Effect of Dexmedetomidine on Postoperative Opioid Consumption and Pain After Major Spine Surgery. Anesth Analg 122: 1646-1653.

9. Peng K, Liu HY, Wu SR, et al. (2015) Effects of Combining Dexmedetomidine and Opioids for Postoperative Intravenous Patient-controlled Analgesia: A Systematic Review and Metaanalysis. Clin J Pain 31: 1097-1104.

10. Lewis SR, Nicholson A, Smith AF, et al. (2015) Alpha-2 adrenergic agonists for the prevention of shivering following general anaesthesia. Cochrane Database Syst Rev.

11. Alfonsi P (2003) Postanaesthetic shivering. Epidemiology, pathophysiology and approaches to prevention and management. Minerva Anestesiol 69: 438-442.

12. Frank SM, Fleisher LA, Breslow MJ, et al. (1997) Perioperative maintenance of normothermia reduces the incidence of morbid cardiac events. A randomized clinical trial JAMA 277: 1127-1134.

13. Alfonsi P (2001) Postanaesthetic shivering: Epidemiology, pathophysiology, and approaches to prevention and management. Drugs 61: 2193-2205.

\section{DOI: $10.36959 / 377 / 331$}

